\title{
A seismic-shielding structure based on phononic crystal
}

Julin Wang

Institute of Building Structures, Shanxi Architectural College

XueFu Street, 030006. Taiyuan (China)

wangjl_tj@163.com

https://orcid.org/0000-0003-0820-9725

Manuscript Code: 13756

Date of Acceptance/Reception: 17.11.2020/14.03.2020

DOI: $10.7764 /$ RDLC.19.3.272

\section{Abstract}

In this paper, a seismic-shielding structure is presented based on the phononic crystal theory, which is now used to control acoustic waves. An earthquake-proof barrier of seismic waves can be built by filling up the structure under the ground around the building that we want to protect. When the frequency of seismic waves falls into the band gaps of the structure, the seismic wave can be blocked. Herein, the frequency band gaps of the structure are determined theoretically, and the influence of geometric and material parameters on the frequency band gaps is analyzed.

Key words: seismic waves; vibration isolation; band gaps

\section{Introduction}

Of all the possible natural hazards, earthquakes are among the most catastrophic. Traditional approaches of protecting buildings are based on vibration isolation of structures, which cause a shift in the fundamental vibration frequency of structures (Spencer \& Nagarajaiah, 2003). In general, these approaches are a little less efficient for large earthquakes and ignore soil-foundation interactions that play a key role in the overall earthquake response of buildings (Marco, Anastasiia, Federico, \& Nicola, 2016). Recently proposed seismic wave barriers made of mechanical metamaterials can significantly reduce seismic wave energy in certain frequency ranges, which provide the means to protect distributed areas rather than individual structures. Based on the phononic crystal theory, their strategy is that seismic waves can be attenuated before they reach protected targets, and so would be largely preferable (Shi \& Huang, 2013).

Liu et al. (2000), fabricated sonic crystals with unconventional properties such as frequency band gaps (BGs), i.e. frequency ranges within which wave propagation is inhibited regardless of the incidence angle of an incoming wave. Following the ongoing phononic crystal works on solid materials, for earthquake protection purposes, the concept of attenuating of the seismic wave by using band gaps of seismic crystal structures has been initially proposed by Baris Baykant \& Serkan (2011). In their study, seismic metamaterials were initially suggested and can attenuate seismic waves at their band gaps. Based on the characteristics of phononic crystals, suitable materials are selected to form crystal unit cells, and the unit cells go to a larger scale and are precisely arranged to generate seismic metamaterial (SM) ( Krodel, Thomé, \& Daraio, 2015). The SM is arranged around buildings with a certain depth and width, as shown in Figure 1 (Sang-Hoon \& Mukunda, 2012). When the seismic wave propagates, the corresponding frequency wave falls into the frequency band gaps of the metamaterial and cannot continue to propagate (Brûlé, Javelaud, Enoch, \& Guenneau, 2014).

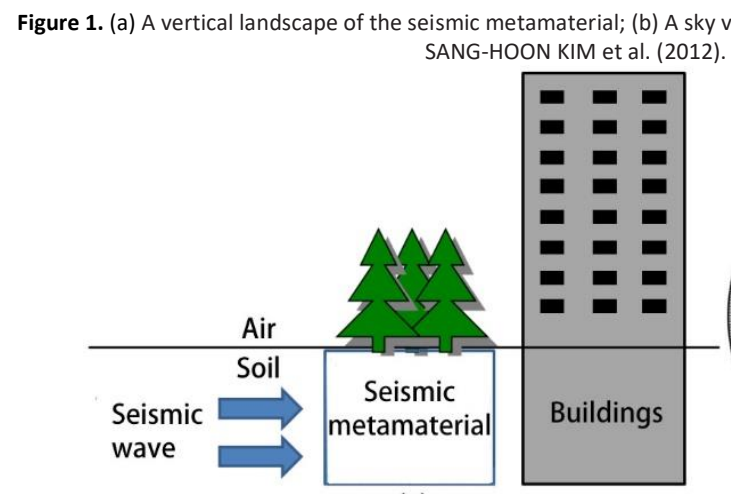

(a)

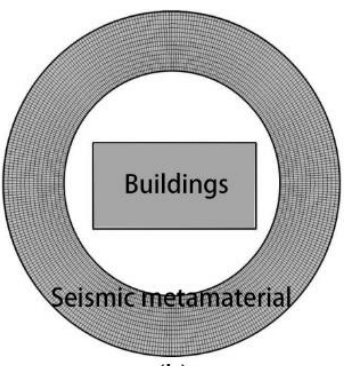

(b)

Brûlé et al. simulated the structural characteristics of metamaterials by considering the entire drilling area as a giant metamaterial structure, which plays the role of isolating and reducing vibration as shown in Figure 2 . In the experiment, 
the design of the SM model is relatively simple, it can only filter seismic waves of a certain frequency. Moreover, the effect of spatial differences in soil physical properties on both seismic wave and BGs was not considered. SANG-HOON et al. (2012) constructed a cylindrical shell-type waveguide composed of many Helmholtz resonators that created a stop-band for the seismic frequency range, which converted the seismic wave into an attenuated one without touching the building expected to be protected as shown in Figure 3. Finocchio et al. (2014) introduced a SM which is composed by a chain of mass-in-mass system able to filter the S-waves of an earthquake. The SM modified the soil behavior, and in the presence of an internal damping, the amplitude of the soil amplification function was reduced also in a region near the resonance frequency. Field experiments of a SM served as the foundation of upper structures were conducted (Yan et al., 2014). The test results showed the foundation can effectively reduce the response of the upper structures for excitations with frequencies within the frequency band gaps.

Figure 2. (a) The cross section of testing device in the x-z plane; (b) Photograph of the seismic metamaterial experiment. Source: S. Brûlé et al. (2014).

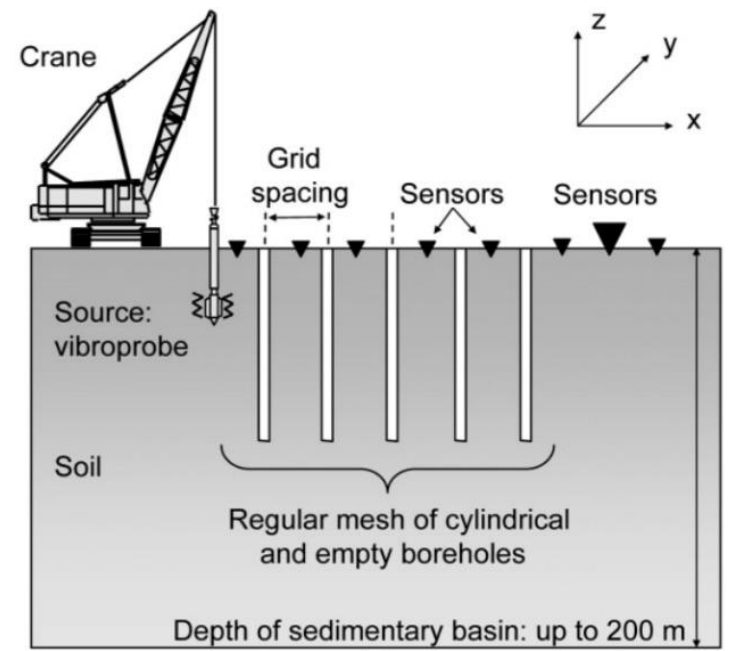

(a)

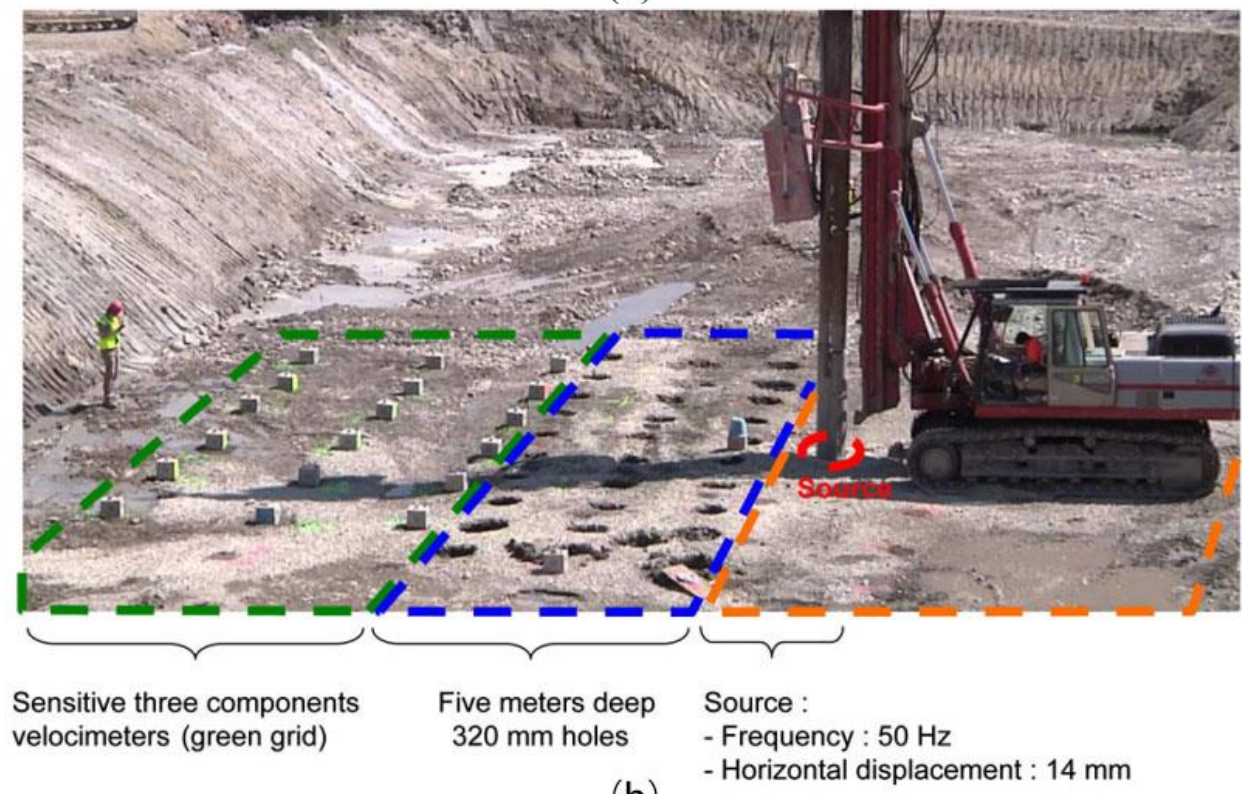

(b)

Figure 3. (a) A sample of a meta-cylinder with four side holes; (b) A combined form of the four meta-cylinders. Source: SANG-HOON KIM et al. (2012).

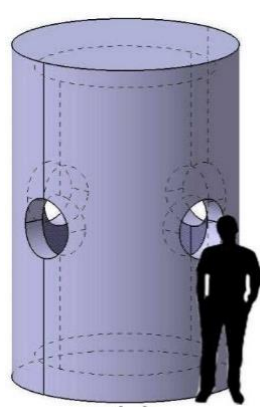

(a)

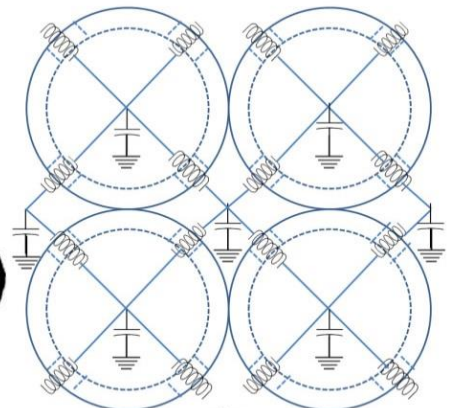

(b) 
A SM was proposed to be buried around sensitive buildings to control the propagation of seismic waves (Krödela, Thoméa, \& Daraio, 2015), which consisted of arrays of cylindrical tubes containing a resonator suspended by soft bearings. Yan et al. (2015) studied the basic theory of three dimensional periodic foundations made up of a SM as shown in Figure 4, and tested the presented foundation with a superstructure in the field. The test results showed that the response of the upper structure was reduced under excitation waves with the main frequency falling in the attenuation zones.

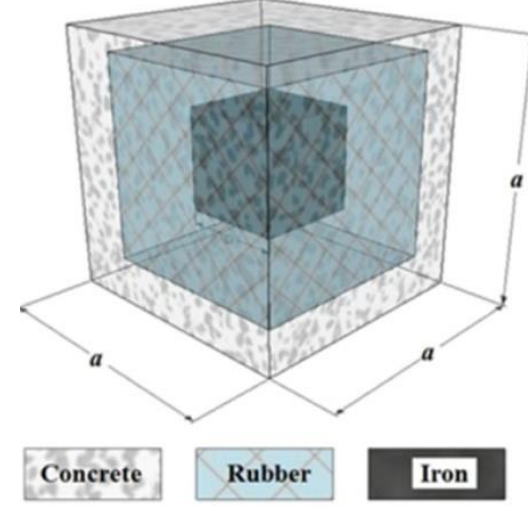

(a)

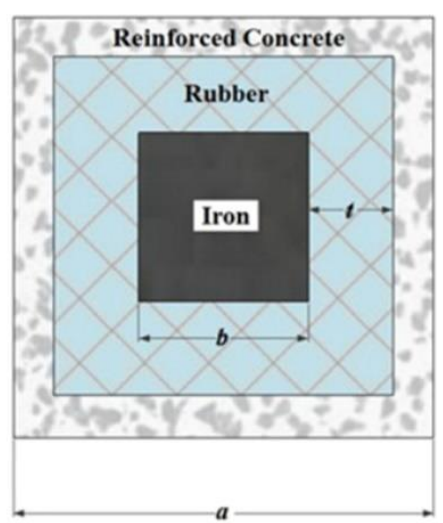

(b)

In this paper, a seismic-shielding structure is proposed, which is buried around protected buildings. In order to investigate the vibration isolation performance of the structures, the spectral element method is adopted for the dynamics modeling, and the corresponding vibration are further analyzed. Furthermore, the influence of both geometric and mechanical parameters on the attenuation potential is analyzed.

\section{Basic Theory}

The seismic-shielding structure proposed in the paper is shown in the Figure 5. Each column of the structure contains $N$ unit cells, and each unit cell is composed of material A and material B, as shown in Figure 6. For a geometrically and materially uniform simple structure, it can be considered as only one spectral element in the SEM. For the complex periodic structures containing a big amount of such simple structures, the element number of the complex structures can be reduced largely in the SEM, the degree of freedom can be also reduced and the computational cost can be decreased. On the other hand, the SEM can obtain highly accurate results in the frequency domain, which is very convenient for the band-gap analysis in the frequency domain. Firstly, the spectrum stiffness matrix is set up for the plates of a unit cell, and the equation of motion of the seismic-shielding structure will be established in the global coordinate system. By analyzing and solving the equation of motion, the frequency response curve of each point is obtained. Finally, the response in the frequency domain is analyzed to study the vibration behavior and vibration isolation of the structure.

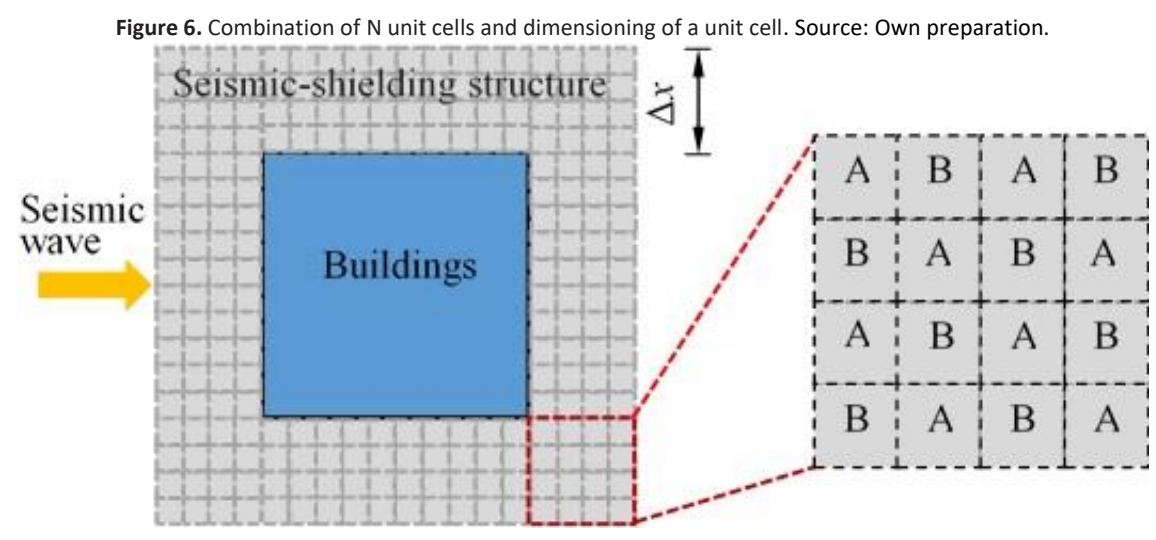


Figure 5. Top view of the proposed seismic-shield structure and layout of unit cells.

Source: Own preparation.
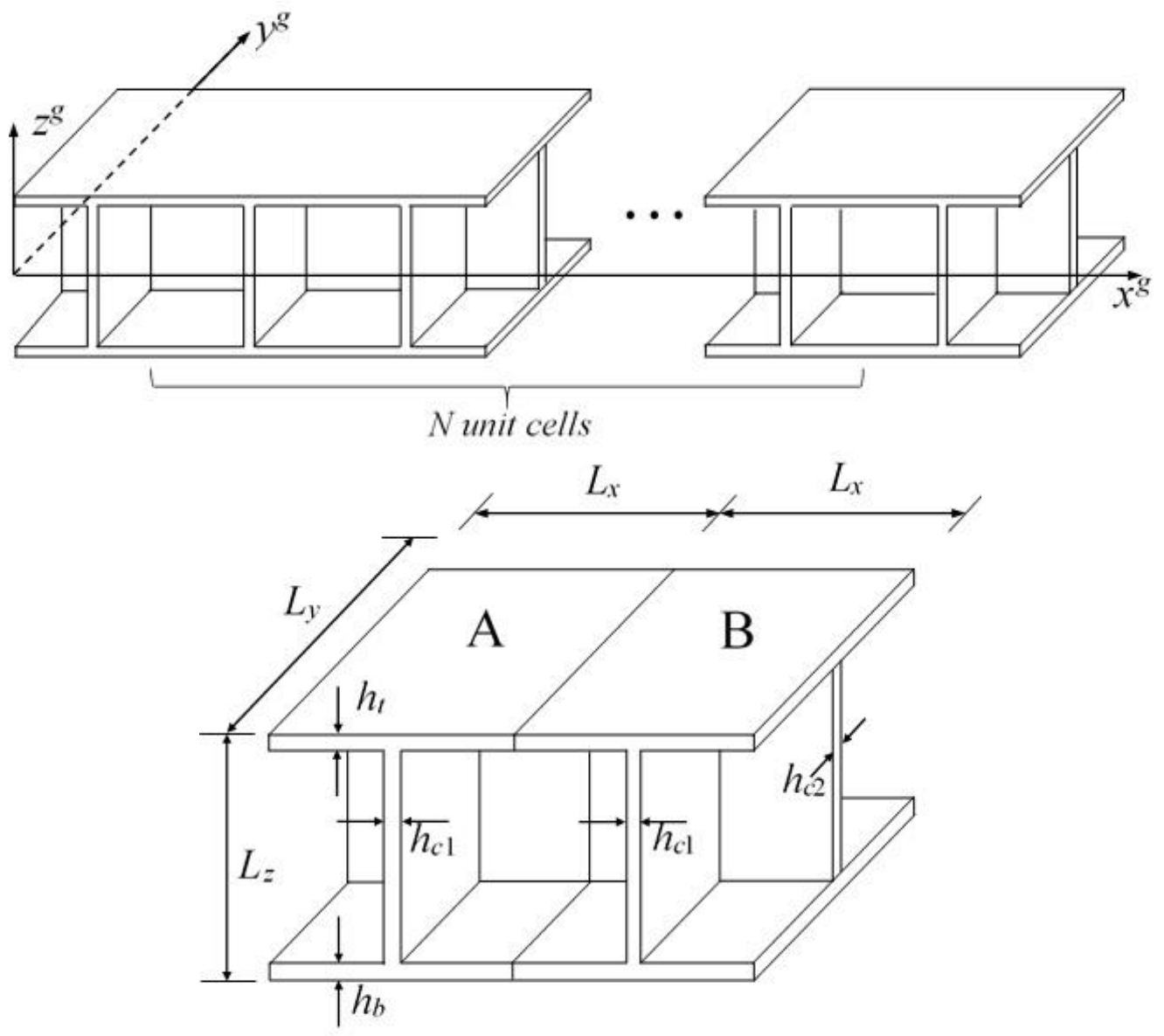

A unit cell

\section{Spectral dynamic equation of plate element}

The thickness of the plates constituting the seismic-shielding structure is much smaller than their length and width, so the effects of shear and moment of inertia are negligible, and the deformed cross section is still perpendicular to the neutral plane. Based on the above assumptions, the plates are Kirchhoff ones.

\section{Out-of-plane vibration}

Figure 7 shows a simple-supported Kirchhoff plate with opposite sides, which is $L$ long, $b$ wide, and $h$ thick. The bending vibration equation of Kirchhoff plates is expressed as:

$$
D \nabla^{4} w(x, y, t)+\rho h \frac{\partial^{2} w(x, y, t)}{\partial t^{2}}=0
$$

where $w(x, y, t)$ is the displacement in the z direction; $\rho$ is the density; $D$ is the bending stiffness of the plate, which can be expressed as: $D=E h^{3} /\left[12\left(1-v^{2}\right)\right]$, where $E$ is Young 's Modulus, $v$ is Poisson's ratio.

According to Figure 8, the shear force and bending moment of the plate are 


$$
\left\{\begin{array}{l}
Q_{x}=-D\left(\frac{\partial^{3} w}{\partial x^{3}}+(2-v) \frac{\partial^{3} w}{\partial x \partial y^{2}}\right) \\
M_{x}=-D\left(\frac{\partial^{2} w}{\partial x^{2}}+v \frac{\partial^{2} w}{\partial y^{2}}\right) \\
Q_{y}=-D\left(\frac{\partial^{3} w}{\partial y^{3}}+(2-v) \frac{\partial^{3} w}{\partial y \partial x^{2}}\right) \\
M_{y}=-D\left(\frac{\partial^{2} w}{\partial y^{2}}+v \frac{\partial^{2} w}{\partial x^{2}}\right)
\end{array}\right.
$$

Figure 7. Simple-supported plate with opposite sides. Source: Own preparation.
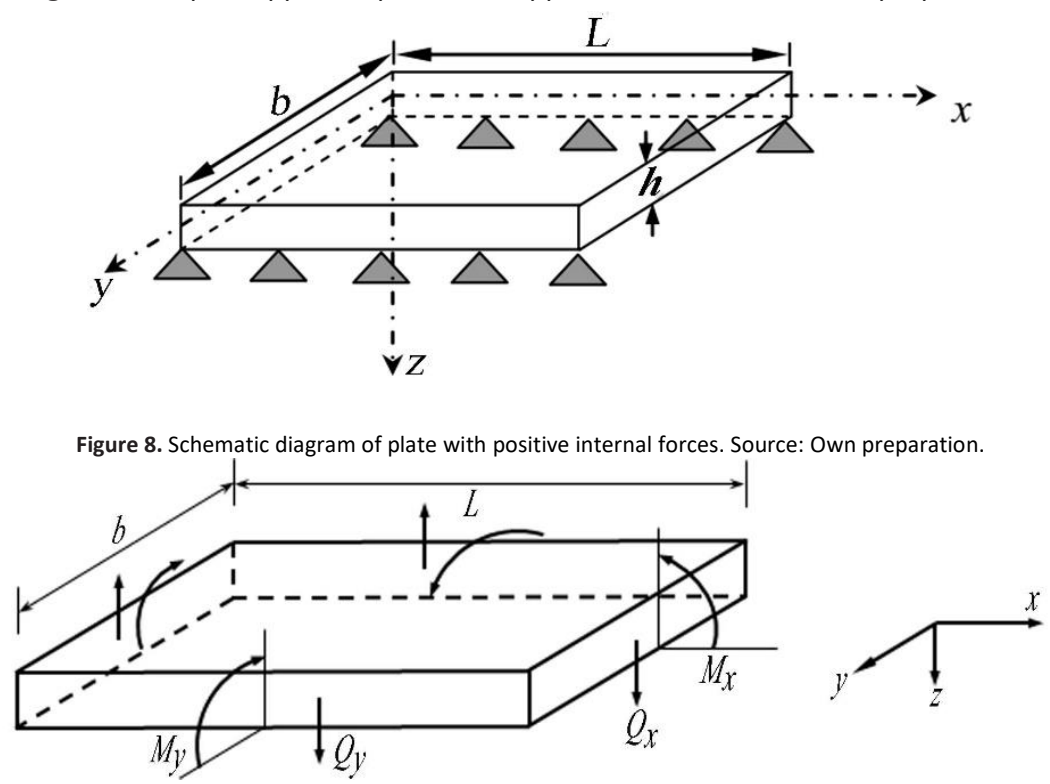

We can obtain the solution of Eq. (1) using Spectral Element Method (Lee, 2009).

$w(x, y, t)=\frac{1}{S} \sum_{n=0}^{S-1} W_{n}\left(x, y ; \omega_{n}\right) e^{i \omega_{n} t}$

where $\omega_{n}$ is the circular frequency; $W_{n}\left(x, y ; \omega_{n}\right)$ is the spectral component of the displacement $w(x, y, t) ; S$ is the number of samples in the time domain.

Substituting Eq. (3) into Eq. (1), we can get

$\nabla^{4} W_{n}-\Omega_{n}^{4} W_{n}=0$

where $\boldsymbol{\Omega}_{n}^{2}=\omega_{n} \sqrt{\rho h / D}$.

Substituting Eq. (3) into Eq. (2), the spectral components of shear force and bending moment can be obtained: 


$$
\left\{\begin{array}{l}
Q_{x n}=-D\left(\frac{\partial^{3} W_{n}}{\partial x^{3}}+(2-v) \frac{\partial^{3} W_{n}}{\partial x \partial y^{2}}\right) \\
M_{x n}=-D\left(\frac{\partial^{2} W_{n}}{\partial x^{2}}+v \frac{\partial^{2} W_{n}}{\partial y^{2}}\right) \\
Q_{y n}=-D\left(\frac{\partial^{3} W_{n}}{\partial y^{3}}+(2-v) \frac{\partial^{3} W_{n}}{\partial y \partial x^{2}}\right) \\
M_{y n}=-D\left(\frac{\partial^{2} W_{n}}{\partial y^{2}}+v \frac{\partial^{2} W_{n}}{\partial x^{2}}\right)
\end{array}\right.
$$

The solution of Eq. (4) has the following form:

$W_{n}\left(x, y ; \omega_{n}\right)=X_{n}\left(x ; \omega_{n}\right) Y_{n}(y)$

For a rectangular plate as shown in Figure 7, it is simply supported along the two boundaries of $y=0$ and $y=b$. The boundary conditions can be expressed as:

$$
\left\{\begin{array}{cc}
w(x, 0, t)=0 ; & w(x, b, t)=0 \\
M_{y}(x, 0, t)=0 ; & M_{y}(x, b, t)=0
\end{array}\right.
$$

Substituting Eq. (3) into Eq. (7), we can get:

$$
\left\{\begin{array}{cc}
W_{n}\left(x, 0, \omega_{n}\right)=0 ; & W_{n}\left(x, b, \omega_{n}\right)=0 \\
M_{y n}\left(x, 0, \omega_{n}\right)=0 ; & M_{y n}\left(x, b, \omega_{n}\right)=0
\end{array}\right.
$$

Substituting Eq. (6) into Eq. (8), we obtain:

$$
\left\{\begin{array}{cc}
Y_{n}(0)=0 ; & Y_{n}(b)=0 \\
Y_{n}^{\prime \prime}(0)=0 ; & Y_{n}^{\prime \prime}(b)=0
\end{array}\right.
$$

In order to satisfy Eq. (9), $Y_{n}(y)$ can be expressed as follows:

$$
Y_{n}(y)=\sum_{m=1}^{s} \sin \left(k_{y n m} y\right)
$$

where $s$ is the number of modes in the y direction; $k_{y n m}=\frac{m \pi}{6} \quad(m=1,2 \cdots s)$ is the wavenumber. Substituting Eq. (10) into Eq. (6), $W_{n}\left(x, y ; \omega_{n}\right)$ can be expressed as:

$$
W_{n}\left(x, y ; \omega_{n}\right)=\sum_{m=1}^{s} W_{n m}\left(x ; k_{y n m}, \omega_{n}\right)
$$

where $W_{n m}$ is the displacement in the wavenumber domain, which has the following form: 
$W_{n m}\left(x ; k_{y n m}, \omega_{n}\right)=X_{n m}\left(x ; k_{y n m}, \omega_{n}\right) \sin \left(k_{y n m} y\right)$

The above equation has a stable simple harmonic solution, and $X_{n m}$ can be expressed as $X_{n m}=\bar{c}_{n m} e^{p x}$, substituting into Equation (11), we obtain:

$W_{n}\left(x, y ; \omega_{n}\right)=\sum_{m=1}^{s} \bar{c}_{n m} e^{p x} \sin \left(k_{y n m} y\right)$

Substituting Eq. (13) into Eq. (4), we get the following equation where $p$ is unknown:

$$
p^{4}-2 k_{y n m}^{2} p^{2}+k_{y n m}^{4}-\Omega_{n}^{4}=0
$$

Solving the above equation, we can get:

$$
\left\{\begin{array}{cc}
p_{1}=\sqrt{\Omega_{n}^{2}+k_{y n m}^{2}} ; & p_{2}=\sqrt{-\Omega_{n}^{2}+k_{y n m}^{2}} \\
p_{3}=-\sqrt{\Omega_{n}^{2}+k_{y n m}^{2}} ; & p_{4}=-\sqrt{-\Omega_{n}^{2}+k_{y n m}^{2}}
\end{array}\right.
$$

Let $c_{n m}=\bar{c}_{n m} \sin \left(k_{y n m} y\right)$, Eq. (12) is transformed into the following form:

$W_{n m}=c_{n m} e^{p x}$

$W_{n m}$ is represented by four values of $p$, and the above equation can be expressed as:

$W_{n m}=\mathbf{E}_{n m} \mathbf{c}_{n m}=c_{n m 1} e^{p_{1} x}+c_{n m 2} e^{p_{2} x}+c_{n m 3} e^{p_{3} x}+c_{n m 4} e^{p_{4} x}$

where $\mathbf{E}_{n m}=\left[e^{p_{1} x} e^{p_{2} x} e^{p_{3} x} e^{p_{4} x}\right], \mathbf{c}_{n m}=\left[c_{n m 1} c_{n m 2} c_{n m 3} c_{n m 4}\right]^{T}$

The rotation angle $\Theta_{x n}$ in the frequency domain can be obtained from $\Theta_{x n}=-\frac{\partial W_{n}\left(x, y ; \omega_{n}\right)}{\partial x}$.

As shown in Figure 9, Node 1 and 2 are at the boundaries of $x=0$ and $x=L$, respectively. The nodal displacements in the frequency domain can be expressed as:

$$
\mathbf{d}_{n}\left(\omega_{n}\right)=\left[\begin{array}{c}
W_{1 n}\left(y ; \omega_{n}\right) \\
\Theta_{1 n}\left(y ; \omega_{n}\right) \\
W_{2 n}\left(y ; \omega_{n}\right) \\
\Theta_{2 n}\left(y ; \omega_{n}\right)
\end{array}\right]=\left[\begin{array}{r}
W_{n}\left(0, y ; \omega_{n}\right) \\
-W_{n}^{\prime}\left(0, y ; \omega_{n}\right) \\
W_{n}\left(L, y ; \omega_{n}\right) \\
-W_{n}^{\prime}\left(L, y ; \omega_{n}\right)
\end{array}\right]
$$

where "' represents the partial derivative with respect to $x$. 


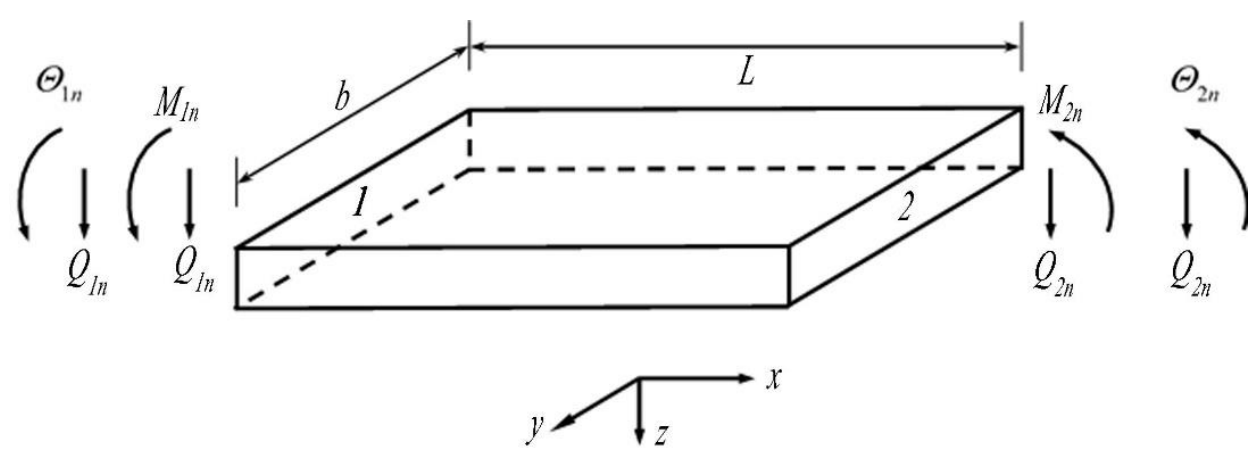

Node displacements and rotation angles in the wavenumber domain can be expressed as:

$\mathbf{d}_{n m}\left(k_{y n m}, \omega_{n}\right)=\left[\begin{array}{c}W_{1 n m}\left(k_{y n m}, \omega_{n}\right) \\ \Theta_{1 n m}\left(k_{y n m}, \omega_{n}\right) \\ W_{2 n m}\left(k_{y n m}, \omega_{n}\right) \\ \Theta_{2 n m}\left(k_{y n m}, \omega_{n}\right)\end{array}\right]=\left[\begin{array}{r}W_{n m}\left(0 ; k_{y n m}, \omega_{n}\right) \\ -W_{n m}^{\prime}\left(0 ; k_{y n m}, \omega_{n}\right) \\ W_{n m}\left(L ; k_{y n m}, \omega_{n}\right) \\ -W_{n m}^{\prime}\left(L ; k_{y n m}, \omega_{n}\right)\end{array}\right]$

Substituting Equation (17) into Equation (19), we can obtain:

$\mathbf{d}_{n m}\left(k_{y n m}, \omega_{n}\right)=\boldsymbol{\Phi}_{n m}\left(k_{y n m}, \omega_{n}\right) \mathbf{c}_{n m}$

where $\boldsymbol{\Phi}_{n m}=\left[\begin{array}{rrrr}1 & 1 & 1 & 1 \\ -p_{1} & -p_{2} & -p_{3} & -p_{4} \\ e^{p_{1} L} & e^{p_{2} L} & e^{p_{3} L} & e^{p_{4} L} \\ -p_{1} e^{p_{1} L} & -p_{2} e^{p_{2} L} & -p_{3} e^{p_{3} L} & -p_{4} e^{p_{4} L}\end{array}\right]$.

According to Eq. (5) and Eq. (17), the shear force and bending moment in the wavenumber domain can be expressed as:

$$
\left\{\begin{array}{l}
Q_{x n m}\left(x ; k_{y n m}, \omega_{n}\right)=-D\left[\mathbf{E}_{n m}^{\prime \prime \prime}(x)-(2-v) k_{y n m}^{2} \mathbf{E}_{n m}^{\prime}(x)\right] \mathbf{c}_{n m} \\
M_{x n m}\left(x ; k_{y n m}, \omega_{n}\right)=-D\left[\mathbf{E}_{n m}^{\prime \prime}(x)-v k_{y n m}^{2} \mathbf{E}_{n m}^{\prime}(x)\right] \mathbf{c}_{n m}
\end{array}\right.
$$

In the wavenumber domain, the shear and bending moments of the nodes are:

$$
\mathbf{f}_{n m}\left(k_{y n m}, \omega_{n}\right)=\left[\begin{array}{c}
Q_{1 n m}\left(k_{y n m}, \omega_{n}\right) \\
M_{1 n m}\left(k_{y n m}, \omega_{n}\right) \\
Q_{2 n m}\left(k_{y n m}, \omega_{n}\right) \\
M_{2 n m}\left(k_{y n m}, \omega_{n}\right)
\end{array}\right]=\left[\begin{array}{c}
Q_{x n m}\left(0 ; k_{y n m}, \omega_{n}\right) \\
-M_{x n m}\left(0 ; k_{y n m}, \omega_{n}\right) \\
Q_{x n m}\left(L ; k_{y n m}, \omega_{n}\right) \\
-M_{x n m}\left(L ; k_{y n m}, \omega_{n}\right)
\end{array}\right]
$$

Substituting Eq. (21) into Eq. (22), we can get: 
$\mathbf{f}_{n m}\left(k_{y n m}, \omega_{n}\right)=\mathbf{G}_{n m}\left(k_{y n m}, \omega_{n}\right) \mathbf{c}_{n m}$

$$
\mathbf{G}_{n m}\left(k_{y n m}, \omega_{n}\right)=-D\left[g_{i j}\right] \quad(i, j=1,2,3,4)
$$

where

$$
\begin{array}{ll}
g_{1 j}=-p_{j}^{3}+(2-v) k_{y n m}^{2} p_{j} ; & g_{2 j}=-p_{j}^{2}+v k_{y n m}^{2} \\
g_{3 j}=p_{j}^{3} e^{p_{j} L}-(2-v) k_{y n m}^{2} p_{j} e^{p_{j} L} ; & g_{4 j}=-p_{j}^{2} e^{p_{j} L}+v k_{y n m}^{2} e^{p_{j} L}
\end{array} .
$$

Solving Eq. (20) gives $\mathbf{c}_{n m}=\boldsymbol{\Phi}_{n m}^{-1}\left(k_{y n m}, \omega_{n}\right) \mathbf{d}_{n m}\left(k_{y n m}, \omega_{n}\right)$, Substituting into Eq. (23), we can obtain:

$\mathbf{S}_{n m}^{K}\left(k_{y n m}, \omega_{n}\right) \mathbf{d}_{n m}=\mathbf{f}_{n m}$

where $\mathbf{S}_{n m}^{K}\left(k_{y n m}, \omega_{n}\right)=\mathbf{G}_{n m}\left(k_{y n m}, \omega_{n}\right) \boldsymbol{\Phi}_{n m}^{-1}\left(k_{y n m}, \omega_{n}\right)$ is the spectral stiffness matrix of the simply supported Kirchhoff plate on the opposite side, which is a matrix related to frequency and wavenumber.

\subsubsection{In-of-plane vibration}

The in-plane vibration of the plate can be described by the following equation:

$$
\left\{\begin{array}{l}
\frac{\partial^{2} u}{\partial x^{2}}+\frac{1-v}{2} \frac{\partial^{2} u}{\partial y^{2}}+\frac{1+v}{2} \frac{\partial^{2} v}{\partial x \partial y}-\frac{\rho\left(1-v^{2}\right)}{E} \frac{\partial^{2} u}{\partial t^{2}}=0 \\
\frac{\partial^{2} v}{\partial x^{2}}+\frac{1-v}{2} \frac{\partial^{2} v}{\partial y^{2}}+\frac{1+v}{2} \frac{\partial^{2} u}{\partial x \partial y}-\frac{\rho\left(1-v^{2}\right)}{E} \frac{\partial^{2} v}{\partial t^{2}}=0
\end{array}\right.
$$

where $u$ and $v$ represent displacements in the $\mathrm{x}$ and $\mathrm{y}$ directions, respectively. The solutions of the above equations have the following spectral expressions:

$$
\left\{\begin{array}{l}
u(x, y, t)=\frac{1}{N} \sum_{n=0}^{N-1} U_{n}\left(x, y ; \omega_{n}\right) e^{i \omega_{n} t} \\
v(x, y, t)=\frac{1}{N} \sum_{n=0}^{N-1} V_{n}\left(x, y ; \omega_{n}\right) e^{i \omega_{n} t}
\end{array}\right.
$$

where $U_{n}$ and $V_{n}$ are the spectral components of $u$ and $v$, respectively, with the expressions

$$
\left\{\begin{array}{l}
U_{n}\left(x, y ; \omega_{n}\right)=\sum_{m=1}^{M} U_{n m}\left(x ; k_{y}, \omega_{n}\right) \sin \left(k_{y} y\right) \\
V_{n}\left(x, y ; \omega_{n}\right)=\sum_{m=1}^{M} V_{n m}\left(x ; k_{y}, \omega_{n}\right) \cos \left(k_{y} y\right)
\end{array}\right.
$$

Substituting into Eq. (26) and Eq. (25), we can get: 


$$
\left\{\begin{array}{l}
U_{n m}^{\prime \prime}-\frac{1-v}{2} k_{y}^{2} U_{n m}-\frac{1+v}{2} k_{y}^{2} V_{n m}^{\prime}+\frac{\left(1-v^{2}\right) \rho}{2} \omega^{2} U_{n m}=0 \\
V_{n m}^{\prime \prime}-\frac{1-v}{2} k_{y}^{2} V_{n m}+\frac{1+v}{2} k_{y}^{2} U_{n m}^{\prime}+\frac{\left(1-v^{2}\right) \rho}{2} \omega^{2} V_{n m}=0
\end{array}\right.
$$

Solving the above equations, we can get the following four eigenvalues:

$$
\left\{\begin{array}{l}
\lambda_{1,2}= \pm \sqrt{k_{y}^{2}-k_{L}^{2}} \\
\lambda_{3,4}= \pm \sqrt{k_{y}^{2}-k_{S}^{2}}
\end{array}\right.
$$

where $k_{L}=\omega \sqrt{\rho\left(1-v^{2}\right) / E}$ and $k_{S}=\omega \sqrt{2 \rho(1+v) / E}$ are the wavenumbers of the in-plane longitudinal wave and shear wave, respectively. $U_{n m}$ and $V_{n m}$ can be expressed as:

$$
\left\{\begin{array}{l}
U_{n m}\left(x ; k_{y}, \omega_{n}\right)=\lambda_{1} B_{1} e^{\lambda_{1} x}+\lambda_{2} B_{2} e^{\lambda_{2} x}+k_{y} B_{3} e^{\lambda_{3} x}+k_{y} B_{4} e^{\lambda_{4} x} \\
V_{n m}\left(x ; k_{y}, \omega_{n}\right)=k_{y} B_{1} e^{\lambda_{1} x}+k_{y} B_{2} e^{\lambda_{2} x}+\lambda_{3} B_{3} e^{\lambda_{3} x}+\lambda_{4} B_{4} e^{\lambda_{4} x}
\end{array}\right.
$$

where $B_{1}, B_{2}, B_{3}$ and $B_{4}$ are unknown coefficients. As shown in Figure $10, U_{1 n m}, V_{1 n m}, U_{2 n m}$, and $V_{2 n m}$ are node displacements, which have the following form in the wavenumber domain:

$\mathbf{d}_{i n}\left(k_{y n m}, \omega_{n}\right)=\left[\begin{array}{c}U_{1 n m}\left(k_{y}, \omega_{n}\right) \\ V_{1 n m}\left(k_{y}, \omega_{n}\right) \\ U_{2 n m}\left(k_{y}, \omega_{n}\right) \\ V_{2 n m}\left(k_{y}, \omega_{n}\right)\end{array}\right]=\left[\begin{array}{c}U_{n m}\left(0 ; k_{y}, \omega_{n}\right) \\ V_{n m}\left(0 ; k_{y}, \omega_{n}\right) \\ U_{n m}\left(L ; k_{y}, \omega_{n}\right) \\ V_{n m}\left(L ; k_{y}, \omega_{n}\right)\end{array}\right]$

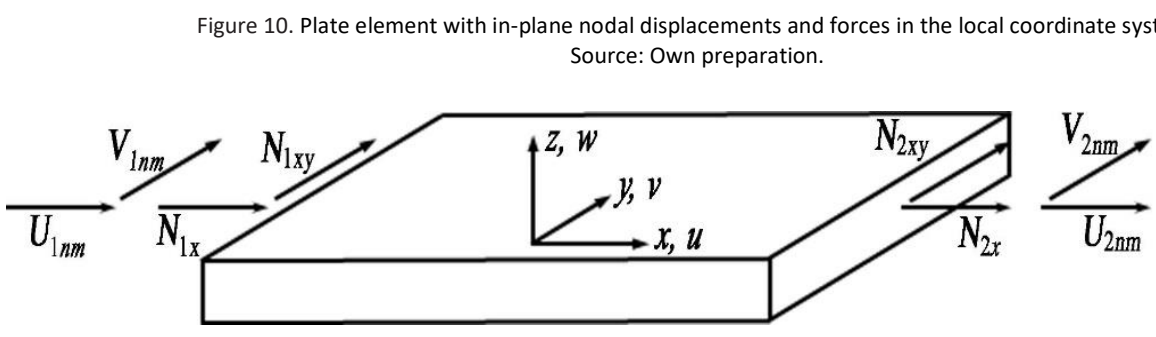

Substituting Eq. (29) into Eq. (30), we can get:

$$
\begin{aligned}
& \mathbf{d}_{i n}\left(k_{y}, \omega_{n}\right)=\boldsymbol{\Phi}_{i n}\left(k_{y}, \omega_{n}\right) \mathbf{B} \\
& \text { where } \boldsymbol{\Phi}_{i n}=\left[\begin{array}{cccc}
\lambda_{1} & \lambda_{2} & k_{y} & k_{y} \\
k_{y} & k_{y} & \lambda_{3} & \lambda_{4} \\
\lambda_{1} e^{\lambda_{1} L} & \lambda_{2} e^{\lambda_{2} L} & k_{y} e^{\lambda_{3} L} & k_{y} e^{\lambda_{4} L} \\
k_{y} e^{\lambda_{1} L} & k_{y} e^{\lambda_{2} L} & \lambda_{3} e^{\lambda_{3} L} & \lambda_{4} e^{\lambda_{4} L}
\end{array}\right], \mathbf{B}=\left[\begin{array}{llll}
B_{1} & B_{2} & B_{3} & B_{4}
\end{array}\right]^{T} .
\end{aligned}
$$


In-plane forces in the frequency and wavenumber domains can be expressed as:

$$
\left\{\begin{array}{l}
N_{x n m}=\frac{E h}{1-v^{2}}\left(\frac{\partial U_{n m}}{\partial x}-v k_{y} V_{n m}\right) \\
N_{x y n m}=\frac{E h}{2(1+v)}\left(k_{y} U_{n m}-\frac{\partial V_{n m}}{\partial x}\right)
\end{array}\right.
$$

As shown in Figure 10, $N_{1 x n m}, N_{1 x y n m}, N_{2 x n m}$, and $N_{2 x y n m}$ are nodal forces, which can be expressed as:

$\mathbf{f}_{i n}\left(k_{y n m}, \omega_{n}\right)=\left[\begin{array}{c}N_{1 x n m}\left(k_{y}, \omega_{n}\right) \\ N_{1 x y n m}\left(k_{y}, \omega_{n}\right) \\ N_{1 x n m}\left(k_{y}, \omega_{n}\right) \\ N_{2 x y n m}\left(k_{y}, \omega_{n}\right)\end{array}\right]=\left[\begin{array}{l}-N_{x n m}\left(0 ; k_{y}, \omega_{n}\right) \\ -N_{x y n m}\left(0 ; k_{y}, \omega_{n}\right) \\ N_{x n m}\left(L ; k_{y}, \omega_{n}\right) \\ N_{x y n m}\left(L ; k_{y}, \omega_{n}\right)\end{array}\right]$

$\mathbf{f}_{\text {in }}\left(k_{y n m}, \omega_{n}\right)$ can also be expressed as:

$\mathbf{f}_{i n}\left(k_{y}, \omega_{n}\right)=\mathbf{G}_{i n}\left(k_{y}, \omega_{n}\right) \mathbf{B}$

where $\mathbf{G}_{i n}\left(k_{y}, \omega_{n}\right)$ is a 4 th order matrix with the following form:

$\mathbf{G}_{i n}\left(k_{y}, \omega_{n}\right)=\left[\begin{array}{cccc}-\alpha\left(\lambda_{1}^{2}-v k_{y}^{2}\right) & -\alpha\left(\lambda_{2}^{2}-v k_{y}^{2}\right) & -\alpha\left(\lambda_{3}^{2}-v k_{y}^{2}\right) & -\alpha\left(\lambda_{4}^{2}-v k_{y}^{2}\right) \\ -2 \beta k_{y} \lambda_{1} & -2 \beta k_{y} \lambda_{2} & -\beta\left(k_{y}^{2}+\lambda_{3}^{2}\right) & -\beta\left(k_{y}^{2}+\lambda_{4}^{2}\right) \\ \alpha\left(\lambda_{1}^{2}-v k_{y}^{2}\right) e^{\lambda_{1} L} & \alpha\left(\lambda_{2}^{2}-v k_{y}^{2}\right) e^{\lambda_{2} L} & \alpha(1-v) k_{y} \lambda_{3} e^{\lambda_{3} L} & \alpha(1-v) k_{y} \lambda_{4} e^{\lambda_{4} L} \\ 2 \beta k_{y} \lambda_{1} e^{\lambda_{1} L} & 2 \beta k_{y} \lambda_{2} e^{\lambda_{2} L} & \beta\left(k_{y}^{2}+\lambda_{3}^{2}\right) e^{\lambda_{3} L} & \beta\left(k_{y}^{2}+\lambda_{4}^{2}\right) e^{\lambda_{4} L}\end{array}\right]$,

$\alpha=E h /\left(1-v^{2}\right), \beta=E h /[2(1+v)]$.

Based on Eq. (31) and Eq. (34), the following equation can be gotten:

$\mathbf{S}_{i n}\left(k_{y}, \omega_{n}\right) \mathbf{d}_{i n}=\mathbf{f}_{i n}$

where $\mathbf{S}_{\text {in }}\left(k_{y}, \omega_{n}\right)=\mathbf{G}_{i n}\left(k_{y}, \omega_{n}\right) \boldsymbol{\Phi}_{i n}^{-1}\left(k_{y}, \omega_{n}\right)$ is the spectral stiffness.

\section{Motion equation of the seismic-shielding structure}

In the small deformation range, the deformation of plates can be decoupled into in-plane and out-of-plane components. Before establishing the motion equation of the structure, the spectral stiffness matrices of in-plane vibration and outof-plane vibration must be assembled to form the complete spectral stiffness matrix of the Kirchhoff plate. Based on the Eq. (24) and Eq. (35), we can get:

$\mathbf{S}_{p}\left(k_{y}, \omega_{n}\right) \mathbf{d}_{p}=\mathbf{f}_{p}$ 
where $\mathbf{d}_{p}$ and $\mathbf{f}_{p}$ are the nodal displacements vector and nodal force vector of the plate, respectively; $\mathbf{S}_{p}$ is the spectral stiffness matrix of Kirchhoff plates including in-plane and out-of-plane vibration components.

The stiffness matrix of Eq. (36) is obtained in the local coordinate system. Since the structure is a spatial structure, the position of the plates in space needs to be described in the global coordinate system. Figure 11 shows the plate element in the local coordinate system and the global coordinate system. The relationship between the local coordinate system and the global coordinate system is as follows:

$$
\left\{\begin{array}{l}
x \\
y \\
z \\
\theta
\end{array}\right\}=\left[\begin{array}{cccc}
\cos \varphi & 0 & \sin \varphi & 0 \\
0 & 1 & 0 & 0 \\
-\sin \varphi & 0 & \cos \varphi & 0 \\
0 & 0 & 0 & 1
\end{array}\right]\left\{\begin{array}{l}
x^{g} \\
y^{g} \\
z^{g} \\
\theta^{g}
\end{array}\right\}
$$

Figure 11. Plate element in the local and global coordinate systems. Source: Own preparation.

Figure 12. A or B of a unit cell divided into twelve spectral plate elements. Source: Own preparation.

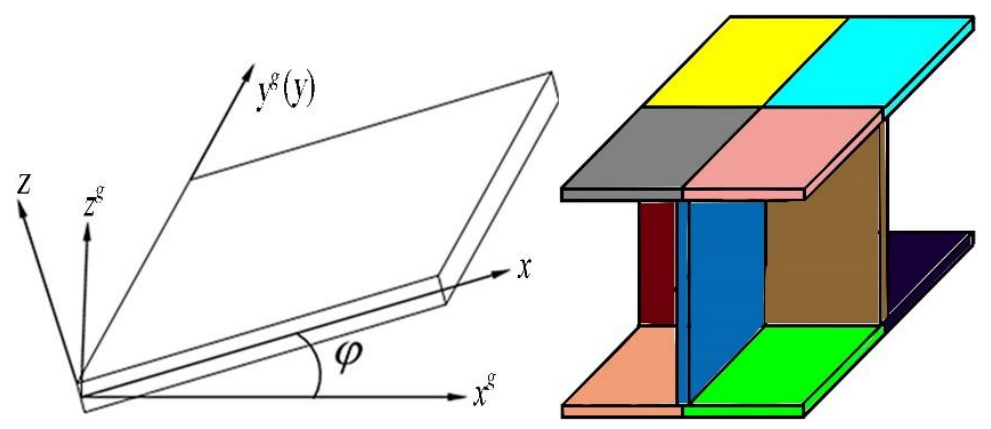

Nodal displacements and nodal force vectors can be transformed from the global coordinate system to the local coordinate system:

$$
\left\{\begin{array}{l}
\mathbf{d}_{p}=\mathbf{T}_{r} \mathbf{d}_{p}^{g} \\
\mathbf{f}_{p}=\mathbf{T}_{r} \mathbf{f}_{p}^{g}
\end{array}\right.
$$

where $\mathbf{d}_{p}^{g}$ and $\mathbf{f}_{p}^{g}$ are the nodal displacements and nodal force vectors in the global coordinate system, and $\mathbf{T}_{r}=\left[\begin{array}{cc}\boldsymbol{\Lambda} & 0 \\ 0 & \boldsymbol{\Lambda}\end{array}\right]$ is the transformation matrix. $\boldsymbol{\Lambda}$ is equal to:

$$
\boldsymbol{\Lambda}=\left[\begin{array}{cccc}
\cos \varphi & 0 & \sin \varphi & 0 \\
0 & 1 & 0 & 0 \\
-\sin \varphi & 0 & \cos \varphi & 0 \\
0 & 0 & 0 & 1
\end{array}\right]\left\{\begin{array}{l}
x^{g} \\
y^{g} \\
z^{g} \\
\theta^{g}
\end{array}\right\}
$$

Substituting Eq. (38) into Eq. (36), the spectral stiffness matrix of the plate in the global coordinate system can be obtained:

$$
\mathbf{S}_{p}^{g}=\mathbf{T}_{r}^{T} \mathbf{S}_{p} \mathbf{T}_{r}
$$


where $\mathbf{S}_{p}^{g}$ is the spectral stiffness matrix of the plate in the global coordinate system.

When using spectral element method, A or B of a unit cell can be divided into twelve spectral plate elements as shown in Figure 12. After obtaining the equation of motion of the unit cell, the unit cell can be integrated into the overall structure to establish the equation of motion of the structure, which has the following form:

$$
\mathbf{S}_{w}\left(k_{y}, \omega_{n}\right) \mathbf{d}_{w}=\mathbf{f}_{w}
$$

where $\mathbf{S}_{w}$ is the spectral stiffness matrix of the structure in the global coordinate system; $\mathbf{d}_{w}$ and $\mathbf{f}_{w}$ are the vector of the node displacement and the vector of the node force of the structure, respectively. By solving Eq. (40), the displacement response of each node of the structure in the frequency domain can be obtained, and the vibration isolation of the structure can be analyzed.

\section{Investigation on the vibration isolation}

\section{Calculating the width of the seismic-shielding structure}

The common form of the magnitude, $M$, is the Richter-scale defined by comparing the two amplitudes in a logarithmic scale as (Sang-Hoon et al., 2012):

$$
M=\log \frac{A_{\text {max }}}{A_{0}}
$$

where $A_{\max }$ is the maximum amplitude of the seismic wave received by the earthquake of interest 100 kilometers away from the epicenter, and the unit is $\mu \mathrm{m} ; A_{0}$ is the maximum amplitude of the seismic wave of a magnitude 0 earthquake received 100 kilometers away from the epicenter, and the unit is $\mu \mathrm{m}$.

A seismic wave of wavelength $\lambda$ propagates in $x$ direction, and the amplitude of the wave reduces exponentially as (Villaverde, 2009):

$$
A e^{i k x}=A e^{i 2 \pi n x / \lambda}=A e^{-2 \pi|n| x / \lambda}
$$

where $n$ is the refractive index. Let the initial seismic wave, that is, before entering the seismic-shielding structure, have amplitude $A_{i}$ and magnitude $M_{i}$, and final seismic wave, that is after leaving the seismic-shielding structure, have amplitude $A_{j}$ and magnitude $M_{j}$ following the Eq. (41). Then, $A_{j}$ is written as $A_{i}$ from Eq. (42) as follows:

$$
A_{i} e^{-2 \pi|n| x / \lambda}=A_{j}
$$

We can rewrite Eq. (43) with the definition of the magnitude in Eq. (41) as:

$$
A_{0} 10^{M_{i}} e^{-2 \pi|n| x / \lambda}=A_{0} 10^{M_{j}}
$$

Taking logarithms of both sides of Eq. (44), we can obtain the width of the seismic- shielding structure, $x \rightarrow \Delta x$, as follows:

$$
\Delta x=\frac{\ln 10}{2 \pi} \frac{\lambda \Delta M}{|n|}=\frac{0.366 \lambda}{|n|} \Delta M
$$

where $\Delta M=M_{i}-M_{j}$ 
The wavelength of different seismic waves varies widely, ranging from several kilometers to as short as tens of meters. Herein, the refractive index is $n=2$ and the wavelength of the seismic wave is $\lambda=100 \mathrm{~m}$. We assume Richter magnitude $M$ is reduced from 8 to 5 , that is $\Delta M=3$, and the width of the waveguide surrounding the building is $\Delta x \approx 60 \mathrm{~m}$.

\section{Analyzing frequency BGs of the seismic-shielding structure}

The geometrical parameters are summarized in table 1. The materials for Part A and Part B of the unit cell are steel with (Young's modulus $E_{s}=207 \mathrm{GPa}$, Poisson's ratio $v_{s}=0.3$, mass density $\rho_{s}=7784 \mathrm{~kg} / \mathrm{m}^{3}$ ) and lightweight aggregate concrete with (Young's modulus $E_{l c}=14.9 \mathrm{GPa}$, Poisson's ratio $v_{l c}=0.25$, mass density $\rho_{l c}=1950 \mathrm{~kg} / \mathrm{m}^{3}$ ), respectively. Number of unit cells in each column $N=60 / 6=10$. The external disturbance force $F=F_{0} e^{i \omega t}$ is applied on the left boundary of the seismic-shielding structure and the coordinate of the action point is $(0 \mathrm{~m}, 3 \mathrm{~m}, 3 \mathrm{~m})$, where $F_{0}=5 \mathrm{kN}$. By solving Eq. (40), the frequency domain response of the structure can be obtained. To better illustrate the seismic-shielding structure can effectively attenuate waves in the frequency domain, a Frequency Response Function (FRF) is defined as $20 \log \left(\delta_{j} / \delta_{i}\right)$. The displacement in the $x^{g}$ direction of Node $(0 \mathrm{~m}, 3 \mathrm{~m}, 3 \mathrm{~m})$ and Node $(60 \mathrm{~m}, 3 \mathrm{~m}, 3 \mathrm{~m})$ is denoted by $\delta_{i}$ and $\delta_{j}$, respectively. When $\delta_{j}$ is equal to $\delta_{i}$, the value of FRF equals zero. When $\delta_{j}$ is $31.6 \%$ of $\delta_{i}$, the value of FRF is $-10 \mathrm{~dB}$, which means the seismic-shielding structure significantly reduces the propagation of waves.

As shown in Figure 13, there are 2 frequency BGs $(1.83-3.32 \mathrm{~Hz})$ and $(5.21-7.04 \mathrm{~Hz})$, within which wave propagation is inhibited regardless of the incidence angle of an incoming wave.

\begin{tabular}{ccccccc}
\multicolumn{7}{c}{ Table 1. Geometric parameters used in the calculations (unit: $\mathrm{mm}$ ). Source: Own preparation. } \\
\hline$L_{x}$ & $L_{y}$ & $L_{z}$ & $h_{t}$ & $h_{b}$ & $h_{c 1}$ & $h_{c 2}$ \\
\hline 6000 & 6000 & 6000 & 200 & 200 & 200 & 200 \\
\hline
\end{tabular}

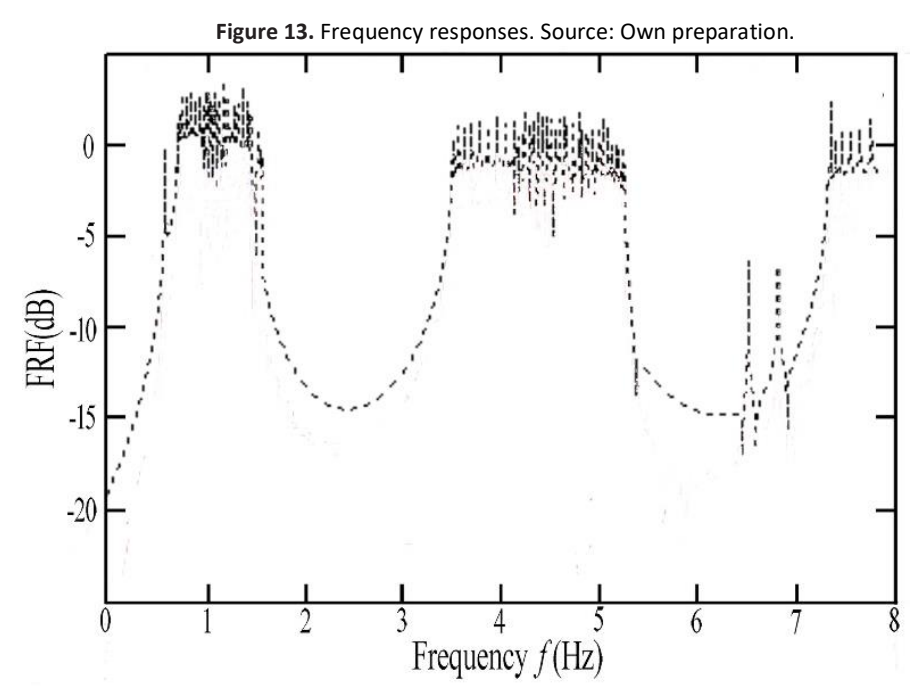

\section{Effects of geometrical parameters on the BG frequencies}

The material and external interference force remain unaltered, and the thickness of the plates is changed as shown in Table 2. Figure 14 shows the frequency response of the seismic-shielding structure when the thickness of the plates changes. It can be seen from the figure that the first BG widens and shifts towards higher frequencies as the thickness of the plate increases. It can be seen that appropriately increasing the thickness of the plate is conducive to improving the effect of low-frequency vibration isolation of the seismic-shielding structure. 


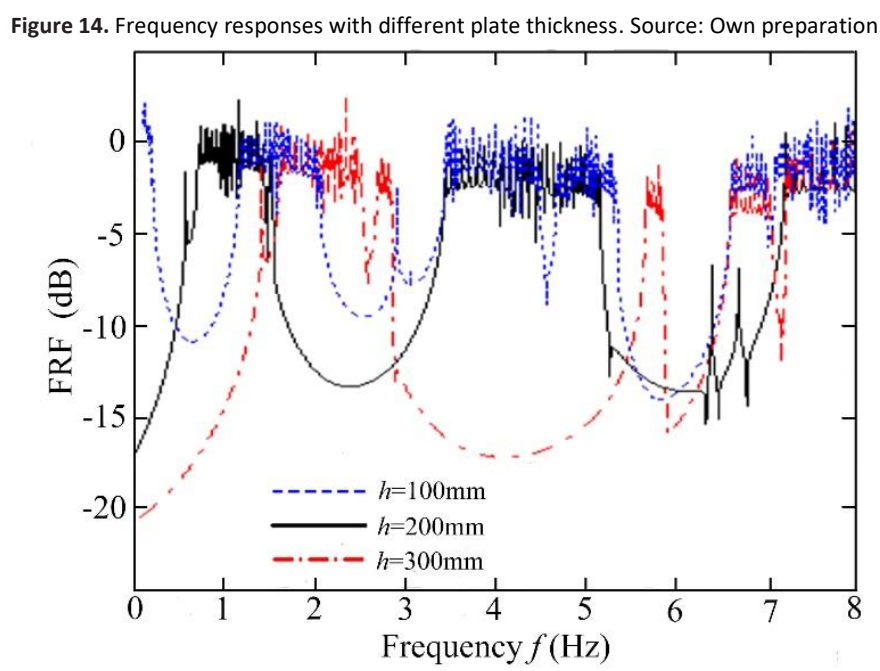

Table 2. Geometric parameters of three cases used in the calculations (unit: $\mathrm{mm}$ ). Source: Own preparation.

\begin{tabular}{ccccc}
\hline Case study & $h_{t}$ & $h_{b}$ & $h_{c 1}$ & $h_{c 2}$ \\
\hline 1 & 100 & 100 & 100 & 100 \\
2 & 200 & 200 & 200 & 200 \\
3 & 300 & 300 & 300 & 300 \\
\hline
\end{tabular}

\section{Effects of material parameters on the BG frequencies}

Next, we analyze the effects of the elastic parameters of the unit cell constituents on the BG frequencies. The material density, Poisson's ratio and Young's modulus are varied, while the size and external interference force remain unaltered to the reference parameters mentioned above. In this section, the materials for the unit cell are lightweight aggregate concrete with (Young's modulus $E_{l c}=14.9 \mathrm{GPa}$, Poisson's ratio $v_{l c}=0.25$, mass density $\rho_{l c}=1950 \mathrm{~kg} / \mathrm{m}^{3}$ ) and concrete with (Young's modulus $E_{c}=30 \mathrm{GPa}$, Poisson's ratio $v_{c}=0.25$, mass density $\rho_{c}=2500 \mathrm{~kg} / \mathrm{m}^{3}$ ), respectively. Figure 15 shows the frequency response of the seismic-shielding structure with two sets of materials. It can be seen from the figure that the two curves are similar, which indicates that material does not play a significant role.

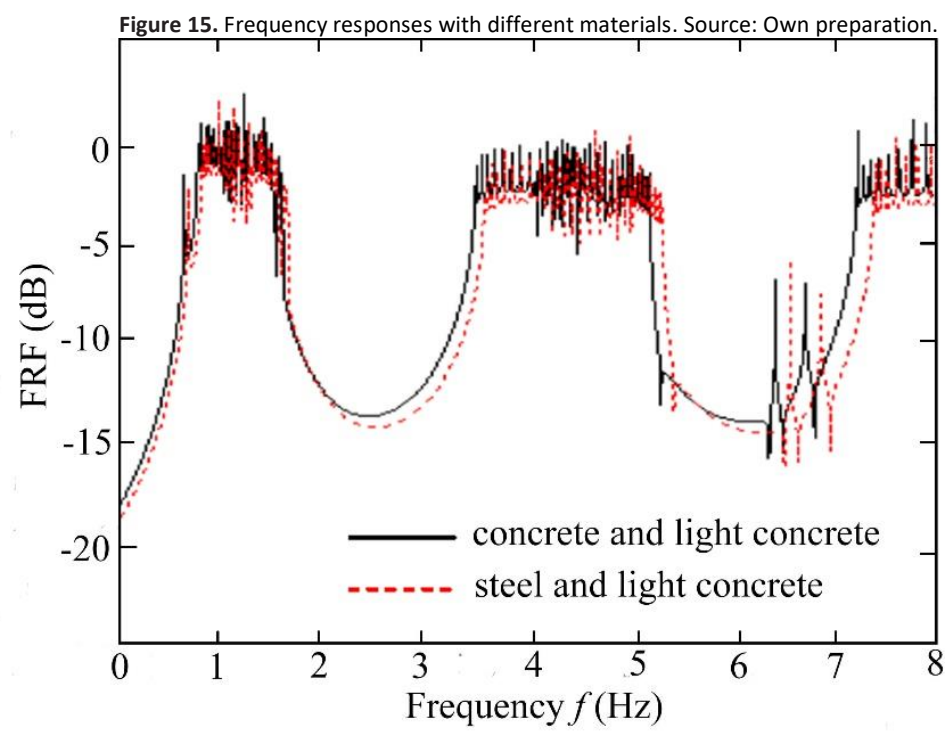


The seismic-shielding structure is presented to reduce the seismic wave energy in the paper. It is not to add another aseismic system to a building but to construct an earthquake-proof barrier around the building to be protected. The proposed structure, contrary to traditional structural foundation isolation strategies, which cause a shift in the fundamental vibrating frequency of civil engineering structures, reduce the seismic wave energy by means of BG attenuation mechanisms and prevent it from reaching the protected site. The structure is composed of steel and lightweight aggregate concrete, making the practical realization of the structure possible from a technical point of view and feasible from an economic point of view when compared to the costs involved with earthquake damage. In the seismic design of structures, this method provides a new idea.

\section{Acknowledgements}

The author would like to acknowledge the support of Dr. Wang Huaning, Tongji University, and Professor Hou Xinlu, Taiyuan University of Technology, for their invaluable support and encouragement.

References

Baris B. A. \& Serkan A. (2011). Towards earthquake shields: A numerical investigation of earthquake shielding with seismic crystals. Open Journal of Acoustics, 1(3), 63-69.

Brûlé S., Javelaud E. H., Enoch S., \& Guenneau S. (2014). Experiments on Seismic Metamaterials: Molding Surface Waves. Physical Review Letters, 112(133901), 1-5.

Finocchio G., Casablanca O., Ricciardi G., Alibrandi U., Garesc F., Chiappini M., \& Azzerboni B. (2014). Seismic metamaterials based on isochronous mechanical oscillators. Applied Physics Letters, 104(191903), 1-5.

Krödela S., Thomé N. \& Daraio C. (2015). Wide band-gap seismic metastructures. Extreme Mech. Lett. 4, 111-117.

Lee U (2009). Spectral Element Method in Structural Dynamics. John Wiley \& Sons, Singapore.

Liu Z. Y., Zhang X. X., Mao Y. W., Zhu Y. Y., Yang Z. Y., Chan C. T., \&Sheng P. (2000). Locally Resonant Sonic Materials. Science, 289, 1734-1736.

Marco M., Anastasiia K., Federico B., \& Nicola M. P. (2016). Large scale mechanical metamaterials as seismic shields. New J. Phys, 18(083041), 1-14.

Sang-Hoon K., Mukunda P. D. (2012). Seismic waveguide of metamaterials. Modern Physics Letters B, 26(17), 1-8.

Shi Z. \& Huang J. (2013). Feasibility of reducing three-dimensional wave energy by introducing perioidc foundations. Soil Dyn. Earthq. Eng. 50, 204212.

Spencer B. \& Nagarajaiah S. (2003). State of the art of structural control. Struct. Eng., 129, 845-856.

Villaverde R. (2009). Fundamental Concepts of Earthquake Engineering. London: Taylor and Francis.

Yan Y., Cheng Z., Meng F., Mo Y. L., Tang Y., \& Shi Z. (2015). Three dimensional periodic foundations for base seismic isolation. Smart Materials and Structures, 24(075006), 1-11.

Yan Y., Laskar A., Cheng Z., Menq F., Tang Y., Mo Y. L., \& Shi Z. (2014). Seismic isolation of two dimensional periodic foundations. Journal of Applied Physics, 116(044908), 1-12. 